\title{
La Denominación de Origen Pisco en Chile: algunos problemas nacionales e internacionales
}

\author{
Pisco Denomination of Origin in Chile: some national and international matters \\ Pablo Lacoste ${ }^{1 *}$, Félix Briones ${ }^{2}$, Diego Jiménez ${ }^{3}$, Bibiana Rendón ${ }^{4}$
}

\begin{abstract}
RESUMEN
El pisco es un aguardiente de uva, elaborado en las regiones de Atacama y Coquimbo, en Chile. Fue delimitado como Denominación de Origen en 1931 y representa la más exitosa DO del Cono Sur de América, con positivos efectos sociales y territoriales. Este artículo examina algunos aspectos sobre el surgimiento de este producto, sus problemas locales y externos.

Palabras clave: pisco, aguardiente, industria vitivinícola, denominaciones de origen.
\end{abstract}

\begin{abstract}
Pisco is a grape brandy, elaborated in Atacama and Coquimbo regions, in Chile. Delimited as Appellation of Origin in 1931, it represents the most successful AO of the South Cone of America, with positive social and territorial effects. This paper examines some aspects about the sprouting of this product, its local and external problems.

Key words: pisco, grape brandy, wine growing industry, appellations of origin.
\end{abstract}

\section{Introducción}

El pisco representa la principal DO del Cono Sur de América. Aguardiente de uva de las regiones de Atacama y Coquimbo, el pisco fue delimitado oficialmente en Chile en 1931 pero con una larga tradición histórica que se remonta a la Colonia española. Actualmente el pisco se apoya en 10.000 hectáreas de viña en manos de 3.000 pequeños viticultores, que elaboran 36 millones de litros al año. La mitad de esta producción la aporta la Cooperativa Capel, formada por 1.700 pequeños propietarios. Esta industria representa un buen ejemplo del positivo efecto social que se puede generar a partir de una DO. A pesar de sus ventajas para el desarrollo equilibrado de la nación y sus aportes a la cohesión social, resulta notable que el pisco sea solo un caso excepcional y que no se hayan desarrollado más DO en la región.

El uso de las DO ha generado numerosos conflictos de interés, muchos de los cuales se han ventilado en los tribunales de la Organización Mundial del Comercio (OMC). Los líderes europeos suelen acusar a los empresarios de países periféricos, sobre todo de América Latina, de falsificar las DO para obtener ventajas comerciales por medios ilícitos. En algunos casos, efectivamente, se han producido maniobras y fraudes con estos fines, en el marco de un largo proceso que tomó fuerza a fines del siglo XIX y se mantiene vigente en la actualidad (Ribeiro de Almeida, 2010; Poças Pintão, 2012).

El proceso de construcción, identificación y reconocimiento de las DO da cuenta de un itinerario de profundas implicancias sociales, culturales, económicas y políticas. Resulta notable la riqueza histórica de las denominaciones de origen como Champagne, Burdeos, Oporto, Jerez, Rioja, Rivera del Duero, Priorato, Tokai, Marsala, entre otros (Duhart y Corona Páez, 2010; Celestino y Blánquez, 2013). Esta tendencia, tan ampliamente desarrollada en Europa, se extendió también a América Latina, donde surgieron algunas DO como Tequila (Gómez

\footnotetext{
Instituto de Estudios Avanzados USACH.

Universidad del Bío-Bío.

Instituto de Estudios Avanzados USACH.

Universidad de Chile.

Autor por correspondencia: pablo.lacoste@usach.cl
}

Fecha de Recepción: 6 Agosto, 2013.

Fecha de Aceptación: 31 Diciembre, 2013. 
Arriola, 2009; Hernández López, 2010) y pisco (Cortés, 2005; Huertas, 2004, 2012). De todos modos se nota una notable asimetría en el desarrollo de las DO en Europa y América: Francia tiene más de 700 DO reconocidas internacionalmente, mientras que en Chile el Instituto Nacional de Propiedad Intelectual apenas ha registrado un puñado de casos (Errázuriz, 2010).

La delimitación de la DO Pisco, dispuesta por el presidente Carlos Ibáñez del Campo en 1931, fue el resultado de un largo proceso histórico-cultural, signado por el interés de los viticultores chilenos por elaborar vinos y aguardientes. La vid llegó a Chile en el segundo tercio del siglo XVI con los conquistadores españoles. Encontró un nicho ecológico adecuado y prosperó rápidamente en la región. Pronto se comenzaron a elaborar los vinos $\mathrm{y}$, poco después, aguardientes.

En la zona central de Chile se difundió la costumbre de elaborar aguardientes a partir de borras y orujos. La idea era aprovechar al máximo el producto de la vid para bajar los costos y asegurar la rentabilidad de la industria en tiempos complicados por la falta de caminos, rutas comerciales y redes de distribución. El tema fue documentado detalladamente en registros oficiales, como el padrón de aguardiente de 1777 , tal como se estudia en otra parte (Lacoste et al., 2014).

La vid llegó a la región en el segundo tercio del siglo XVI, traída por los conquistadores españoles. Estos comenzaron a fundar rápidamente las ciudades de Perú (1532) y Chile (1541). Y en ambos territorios comenzaron a cultivar las primeras viñas desde el primer momento y en forma casi simultánea. Tempranamente se fundó La Serena (1544), para facilitar las comunicaciones entre Perú y Chile. Pronto comenzó el cultivo de la vid y la elaboración de vinos y aguardientes en los valles del Copiapó, Huasco, Elqui y Limarí.

Si bien es cierto que el proceso de fundación de ciudades había comenzado antes en Perú (1532) que en Chile (1541), las primeras fuentes que documentan el desarrollo de la vid en ambos países se producen de forma aproximadamente simultánea. Al mismo tiempo que Pedro Cieza de León dejaba constancia en 1548 del cultivo de la vid en el Perú (Huertas, 2004), ya existían antecedentes de la introducción de la planta ya citada en una concesión otorgada por Pedro de Valdivia a Juan Bautista Pastene en 1546 (Cortés, 2005). La uva moscatel, muy valorada para la elaboración de aguardiente, se adaptó muy bien a la zona. Uno de los primeros registros de la uva moscatel en Chile se detectó en 1614, en actas del Cabildo de Santiago (Lacoste et al., 2010). De todos modos, estas variedades alcanzarían una notable adaptación en los valles vitivinícolas de La Serena y valles conexos.

\section{La DO Pisco en el siglo XIX}

El consumo de aguardiente se difundió en Chile en diversas modalidades. A mediados del siglo XIX el viajero francés Gabriel Lafond de Lucy observó la costumbre de beber aguardiente con jugo de limón y azúcar, modalidad que después se conoció en Chile como "Pisco sour". El autor se interesó por observar las costumbres de los chilenos, incluyendo su cultura material, entretenimientos, indumentaria, comidas y bebidas. Identificó al huaso como personaje típico, y entre otras características, señaló: "su bebida favorita es el ponche frío, hecho con aguardiente, limón, agua y azúcar" (Lafond, 1853).

A mediados del siglo XIX Claudio Gay detectó la costumbre campesina chilena de consumir en abundancia el aguardiente, entre otras bebidas, como componentes vitales de la socialización y reforzamiento de lazos familiares durante el desarrollo y celebración de los ritos nupciales. Del mismo modo, el naturalista francés ya citado describió un aspecto esencial de la vida familiar del campesino:

\begin{abstract}
"Sus noches se pasan de una manera harto monótona; las mujeres ocupadas en preparar la cena y los hombres sentados, en la parte exterior de la casa en el verano y en invierno en la cocina, tratando de cosas insignificantes y a veces sin decir una palabra. Al caer la noche, es decir, a la oración, toman su mate, al que son muy aficionados, o bien cuando no hay lo reemplazan por una bebida de agua caliente preparada con azúcar y limón y muchas veces con un poco de aguardiente, $\mathrm{y}$ después todos arrodillados rezan el rosario, haciendo coro a una persona respetable que repite la oración" (Gay, 2009: 111).
\end{abstract}

Por otra parte, la presencia del aguardiente de uva mezclada con agua y limón en la ejecución de tareas por parte de la servidumbre en las haciendas era importante, especialmente en el caso de los arrieros, descrito a continuación: "Antes de amanecer 
todos los arrieros reúnen sus mulas y se apresuran a cargarlas. Esta operación es siempre muy larga a causa de las numerosas piezas de que se compone el aparejo, y puede calcularse que necesitan por lo menos dos horas para cargar cuatro piaras, o lo que es igual unas cuarenta mulas. Mientras dura esta operación, el madrinero cuida de las mulas cargadas y ofrece a cada uno de los arrieros un pocito de mate, preparado por él, o un poco de agua caliente con azúcar y una raja de limón y algunas veces un poco de aguardiente" (Gay, 2009: 146-147).

Como se puede observar, hubo una notable continuidad en el lazo entre este producto y al pueblo chileno. Así lo recogió la literatura chilena a comienzos del siglo XXI, donde el pisco sour se presenta como "bebida nacional" o "trago nacional". Así se refleja en obras de Isabel Allende tales como Mi país inventado (2003).

En el llamado "norte chico" de Chile se configuró la tradición de elaborar aguardiente de mejor calidad a partir de uvas de moscatel. El perfume de estas variedades fue apreciado como un aporte significativo para el aguardiente. Poco a poco, en torno los valles de Elqui, Limarí, Choapa y Huasco, se pusieron en marcha emprendimientos orientados a elaboración de aguardiente con vinos seleccionados a base de uvas escogidas de moscatel y afines, y se comenzó a utilizar la expresión "Pisco" para denominar este aguardiente.

No obstante, existía un serio problema con el registro de marcas de licores, vinos, aguardientes y pisco, y consiste en que la ley de "Privilegios Exclusivos" vigente desde 1840 hasta noviembre de 1874 protegía solo las patentes de invención, no así las marcas registradas (INAPI, 2010). Esto se ajustaba a las disposiciones contenidas en el artículo 152 de la Constitución de 1833, señalando que "Todo autor o inventor tendrá la propiedad exclusiva sobre su descubrimiento, o producción por el tiempo que le concediere la lei; i si ésta exigiere su publicación, se dará al inventor la indemnización competente". A modo de ejemplo, cuando se diseñó el primer ron en Chile, lo patentado fue el "método para hacer Ron en Chile" (INAPI, 2010), no la DO "Ron de Valparaíso".

Este escenario cambió de manera drástica con la entrada de la Sociedad Nacional de Agricultura (SNA), la que impulsó un cambio en las prácticas industriales agrarias y, por medio de sus intelectuales (v.gr., Julio Menadier), también tuvo una influencia considerable en la producción de proyectos de ley sobre propiedad industrial y registro de marcas, dentro de un contexto en el cual las falsificaciones de marcas y productos para aumentar las ventas era una práctica habitual entre los productores agrícolas. Esto llevó a la SNA a preparar un proyecto de ley en 1874, que se convirtió en la Ley de Marcas de Fábricas de 1874 (INAPI, 2010). De este modo, las marcas pasaron a tener seguridad jurídica, y fue el punto de inflexión sobre la materia, especialmente para el registro de vinos, aguardientes y licores en general (INAPI, 2010).

Poco a poco se comenzaron a registrar las primeras marcas de pisco en la década de 1880 . Por un lado se registró el Pisco G de Copiapó (1882) a nombre del viticultor José María Goyenechea. Por otro se anotó el Pisco Ytalia (1883), con la mención a Elqui en la misma etiqueta. Fue iniciativa de don Juan de Dios Pérez de Arce, abuelo de los Munizaga, impulsores de la empresa pisquera "Los Nichos", situada en la localidad de Pisco Elqui, actualmente en actividad. Le fue otorgada "para toda clase de licores espirituosos" (Figura 1).

Los tratados de vitivinicultura comenzaron a incorporar al pisco como un producto específico que merecía un desarrollo explicativo. En 1891 Manuel Rojas publicó la primera edición de su Manual de Viticultura y Vinificación. Allí se estableció la primera definición de Pisco: "aguardiente común, con solo la diferencia de necesitar uva moscatel para que le transmita su propio aroma. No se usarán los orujos para la destilación sino el vino blanco de cuatro meses de elaborado con todo esmero. En todo Chile puede elaborarse el pisco, desde que la uva moscatel madura en todas partes, pero de mejor calidad resulta donde el clima es más cálido y la uva más aromática. Conocido en todo Chile es el Pisco de Locumba (Perú)" (Rojas, 1891: 374).

Resulta notable el relato de este agrónomo chileno. Su visión del pisco significó avances considerables, pues estableció cuatro condiciones para la elaboración del pisco:

a. Debe elaborarse a partir de vino de uva y no a partir de borras y orujos, tal como era común en el centro del país en el siglo XVIII (Lacoste et al., 2014).

b. Reconoce notas de calidad en el producto de base: debe ser vino blanco, de al menos cuatro meses y "elaborado con todo esmero". c. Define por primera vez que el Pisco requiere variedades de uvas determinadas: 


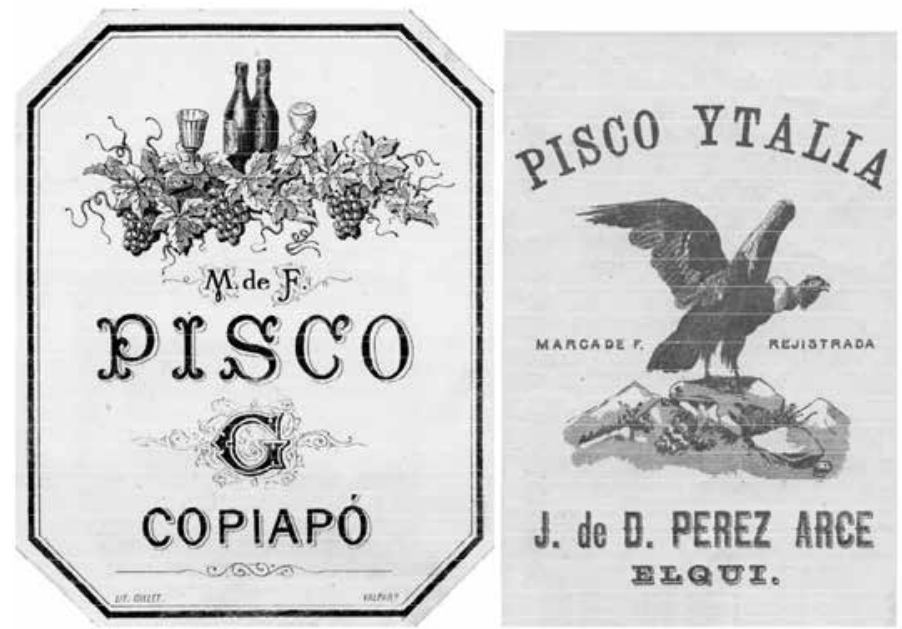

Figura 1. Etiquetas de los primeros piscos registrados bajo Ley de Marcas.

Fuente: Instituto Nacional de Propiedad Industrial. 2010. Historia gráfica de la propiedad industrial en Chile, Santiago de Chile, Pie de Texto Consultores y Editores, p. 33 (Pisco G de Copiapó) y p. 46 (Pisco Ytalia).

\begin{abstract}
"necesita uva moscatel para que le transmita su propio aroma".

d. Insinúa la superioridad de algunas regiones sobre otras para elaborar el pisco: "la mejor calidad resulta donde el clima es más cálido y la uva más aromática”.
\end{abstract}

Para Rojas, el pisco no era, todavía, una DO. Era más bien un genérico que podía elaborarse en cualquier lugar. Señala que "en todo Chile puede elaborarse", como así también en Perú. El enfoque de Rojas estaba marcadamente influido por sus maestros extranjeros, sobre todo René Le Feuvre, que no fueron capaces de admitir que Chile podía desarrollar sus propias DO. Sin embargo, de forma automática el autor detectó que el producto tenía una serie de condiciones y potencialidades propias de un producto de calidad, elaborado con esfuerzo y esmero, y que con preferencia se debía elaborar en determinadas regiones. Con intuición Rojas ya estaba echando las bases para el desarrollo de una denominación de origen.

Los conceptos de Rojas se reiteraron en las ediciones posteriores de esta obra. Por ejemplo, en la de 1950 se reiteran aquellas mismas palabras, con los cuatro elementos que caracterizaban al pisco, a la vez que se mencionaba el cambio aportado por el DFL de 1931: "La ley de alcoholes que delimitó la Región Pisquera establece que el nombre de Pisco queda reservado a los aguardientes que provengan de la destilación de los vinos de uva producidos dentro de la Zona Pisquera" (Rojas, 1950 p.633).

El enfoque de Rojas tuvo un gran efecto en el mundo del vino chileno debido a su posición de prestigio y su capacidad de formación de opinión. Por un lado, Rojas fue un destacado profesor, director de la Escuela de Vitivinicultura de Cauquenes, entre otros cargos. Su Tratado de Viticultura y Vinificación se convirtió en una obra clásica en la región. Fue reeditado numerosas veces. Hacia fines del siglo XX todavía se consideraba su trabajo como la obra más importante que había generado la enología chilena en la materia, y recién fue superada con los libros de Gil y Pszczólkowski (2007) y Pszczólkowski y Ceppi de Lecco (2011).

No obstante, René Le Feuvre, quien fuera en palabras de Rojas "mi maestro", desarrolló informes sobre el aguardiente y el pisco con posterioridad al lanzamiento del tratado del último. Respecto de las variedades de uva intervinientes en la producción de vinos y piscos - primer aspecto clave en una DO-, Le Feuvre señala: "La uva negra común y la uva Mollar forman la base de la generalidad de los viñedos de Vallenar hasta Junta, mientras que en los valles del Carmen y del Tránsito predomina la uva moscatel blanca, llamada uva Italia, que se emplea para la fabricación de la pasa y para obtener el pisco" (Le Feuvre, 1892a). A los varietales de uva ya citados también se añade la moscatel rosada, o uva pastilla para la producción de pisco (Le Feuvre, 1892a). 
Un segundo componente esencial de las DO es la indicación geográfica en la cual se circunscriben las condiciones climáticas, culturales y de suelo para la elaboración de pisco. Al respecto, Le Feuvre indica: "La principal viña del valle de Huasco es la de la Armidita, situada en la plaza del Tránsito, a una altitud de 1.100 metros, aproximadamente. Este hermoso viñedo ha demandado una suma enorme de energía y de voluntad tenaz y gastos considerables a su fundador. Está llamada a dar grandes resultados, una vez que reciba los cuidados necesarios, que se establezcan las industrias de la pasa, del pisco, junto con la fabricación de vinos licorosos y que las bodegas de gobierno de estos vinos se sitúen en el puerto de Huasco, y, en fin, que se haya restablecido el camino carretero para facilitar los transportes hasta Vallenar" (Le Feuvre, 1892a: 354).

En el caso específico de las condiciones climáticas, de cultivo y cuidado de la vid destinada a la producción de pisco, el mismo autor menciona: "En las viñas antiguas el suelo queda inculto, pero generalmente se practica el recorte de los sarmientos y el azuframiento para combatir el oidium. Los viñedos nuevos, sobre todo los que dan la pasa y el pisco, están muy bien cultivados, el cultivo de la planta no deja que desear. Muchas viñas de Paihuano y Monte Grande pueden compararse ventajosamente con los mejores viñedos que se citan como modelos en la región central de la República" (Le Feuvre, 1892a: 406-407).

En relación con las características geográficas que deben reunir las viñas para plantar determinados varietales de uva, Le Feuvre indica, respecto de los viñedos en que se cultivan los diversos varietales de uva en general, y de moscatel en particular, lo siguiente: "Los viñedos están situados en las llanuras bajas de los valles y en las pendientes de las colinas que las limitan. Los primeros se componen principalmente de cepas de uva negra común, Mollar, etc. Los productos que dan sirven para la fabricación de los vinos, de los aguardientes y de la chicha (vino cocido). Las viñas de las colinas están constituidas por la variedad de uva blanca, moscatel, llamada uva Italia, que se emplea en la preparación de las pasas y del aguardiente especial, designado bajo el nombre de pisco" (1892b).

Lo aportado por Le Feuvre es especialmente importante para consolidar las bases fundamentales de una denominación de origen, por cuanto capta gran parte de los aspectos esenciales de las condiciones de desarrollo, cultivo, y producción del aguardiente, el pisco, y los varietales esenciales para su desarrollo, además de sus efectos sociales, económicos y culturales. Además, cabe señalar que varias de las regiones geográficas indicadas por Le Feuvre fueron incluidas en el DFL 181 del 15 de mayo de 1931 que delimita la DO Pisco (Figura 2).

\section{El pisco en la legislación de Chile}

Como toda DO, el desarrollo comercial del pisco requería del reconocimiento y protección por parte del Estado. Ello se concretó en 1931 cuando el presidente Carlos Ibáñez del Campo produjo el Decreto con Fuerza de Ley 181, que se examina más adelante. Pero previo a ello se registraron otras iniciativas vinculadas con el tema, a veces contradictorias, como la Ley No 3.087 de 1916.

Al definir los impuestos que las bebidas alcohólicas debían pagar por litro, la Ley $\mathrm{N}^{\circ} 3.087$ del 13 de abril de 1916, en su artículo único, estableció que el artículo 50 de la Ley $\mathrm{N}^{\circ} 1.515$ del 20 de enero de 1902 prescriba que "los licores con base de vino, oporto, jerez, Málaga y similares pagarán 20 centavos por litro", mientras que los "aguardientes, piscos artificiales, whisky, cognac artificiales, ron, ginebra y similares pagarán 45 centavos por litro". Más adelante se aclara que esta carga solo regía para pisco y cognac artificial, no así para los naturales. Asimismo, el artículo único de la ley ya citada modificó el artículo 52 de la Ley $\mathrm{N}^{\circ} 1.515$ del 20 de enero de 1902, el que a partir de 1916 indica que "se exceptúan del impuesto establecido por el artículo 50 los piscos y cognac naturales, entendiéndose por tales solamente los genuinamente puros que se produzcan directamente de uva especial sin agregados de esencia ni de otras sustancias que las permitidas por el reglamento y que sean embotelladas directamente por el viñero que los produzca".

Junto con la contaminación identitaria representada por el uso de las DO europeas para productos elaborados en Chile, la norma de 1916 incluyó, por primera vez en la legislación nacional, la referencia al aguardiente elaborado en la nación: el pisco. Pero el legislador no fue capaz de reconocerlo como primera $\mathrm{DO}$ de productos espirituosos chilenos ni mucho menos protegerla ni delimitarla. Al contrario, se utilizó el concepto como un genérico más, con el agravante de autorizar la elaboración tanto de piscos naturales como piscos artificiales. 


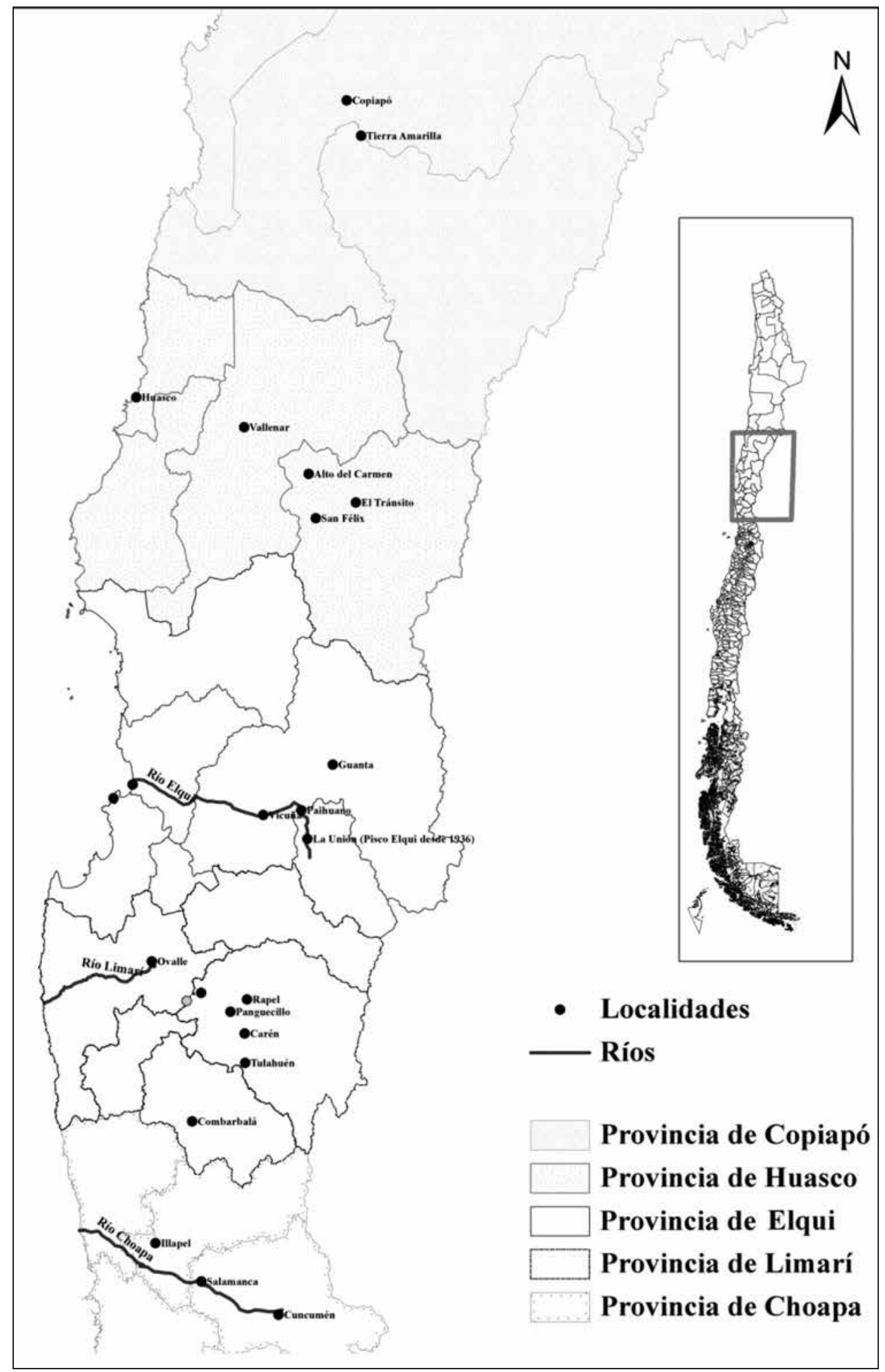

Figura 2. Delimitación de la Denominación de Origen Pisco (1931). 
El Congreso no logró detectar el valor que tenía el pisco para la economía nacional, y lo consideraba como un nombre de fantasía sin mayor relevancia. Para el Estado chileno daba lo mismo usar el nombre "pisco" o "cognac".

En todo caso se establecía una diferencia entre piscos naturales, elaborados a partir de vino de uva, de vinos artificiales, producto obtenido de borras y orujos. Se estableció así un reconocimiento jerárquico de la calidad de los piscos elaborados a partir de vino. Indirectamente se echaron las bases para la evolución posterior.

El ciclo de vigencia del librecambio y la admiración de las grandes potencias del norte como paradigmas indiscutibles para alcanzar el desarrollo se vio severamente afectado por la Gran Depresión económica mundial de 1929. La crisis impulsó a las clases dirigentes de Chile hacia la reflexión, la autocrítica y la redefinición de las posiciones que hasta entonces se habían adoptado en forma casi dogmática. Esta situación se hizo sentir en el campo de los vinos.

En el marco del nuevo paradigma, se produjo la primera delimitación de una DO en el Cono Sur de América. Así se concretó mediante el decretoley 181 del presidente Carlos Ibáñez del Campo (1931) por el cual se estableció el reconocimiento y la protección a la DO Pisco. Se estableció que: "considerando que desde antiguo se elaboran aguardientes en las Zonas de Huasco y Elqui que, con el nombre de Pisco han alcanzado justo renombre en el país y en el extranjero". Asimismo, solo podía usar este nombre el aguardiente de uva destilado en la zona ya definida (departamentos de Copiapó, Huasco, La Serena y Ovalle). Para complementar esta medida, por decreto 5.798 (1936) se impuso el nombre "Pisco Elqui" a la villa de La Unión, situada en el corazón de la zona pisquera, 107 km al este de La Serena, en el centro del valle de Elqui. De esta manera se produjo un promisorio quiebre en el proceso cultural de la vitivinicultura del sur de América, con el surgimiento de la primera DO local.

\section{El pisco y su frustrado reconocimiento en Argentina}

Debido a la fragilidad de las comunidades formadas por los pequeños productores locales, el desarrollo de una DO necesita en forma imperiosa de la protección del Estado, tanto dentro como fuera del país. En el campo interno, el problema del pisco se solucionó con el DFL de 1931. El paso siguiente era obtener el reconocimiento de los demás países. En ese plano se ha avanzado muy poco. Un caso interesante es el de Argentina.

En Argentina, la Gran Depresión también produjo un cambio de paradigma en la percepción de lo que debía ser el rol del Estado frente a la industria de la vid y el vino. Ello se reflejó en la creación de la Junta Reguladora del Vino (Ley No 12.137 de 1935). A partir de entonces se creaba una institucionalidad encargada de producir información estadística sobre la vitivinicultura nacional. Esta organización puso en marcha un proceso sistemático de generar información confiable acerca de la actividad, incluyendo el primer censo vitícola nacional (1936). En este contexto, se preparó una nueva ley de vinos.

La sanción de la Ley $\mathrm{N}^{\circ} 12.372$ (1938) significó un avance considerable en el desarrollo de la industria en general, pero un retroceso en el campo de las DO. Porque a diferencia de lo ocurrido en Chile, el gobierno argentino no fue capaz de sacudirse de la contaminación identitaria de la etapa anterior y, por el contrario, tendió a profundizarla. Esta ley estableció un conjunto de definiciones sobre distintos tipos de vino, donde se oficializó la confusión de Denominaciones de Origen con genéricos. En la página 11.267 de la edición 11.167 del Boletín Oficial la ley ya citada señala: “i) Cognac, aguardiente o pisco se llama solamente el producto proveniente de la destilación especial de vino sano".

Desde el punto de vista conceptual, esta ley consolidó la tendencia de los 40 años anteriores, en el sentido de confundir DO con genéricos. Desde una mirada más específica, esta norma mantuvo la referencia a las DO anteriores (Jerez, Marsala y Oporto), pero agregó otras nuevas que hasta entonces no habían figurado en los cuerpos legislativos de la Argentina: una sobre vinos espumantes, la propuesta por Zeballos, Champagne, y dos referidas al aguardiente: el Cognac francés y el Pisco chileno. Es importante señalar que, para ese entonces, el Estado de Chile ya había delimitado la DO Pisco, la que fue ignorada por el gobierno de Argentina.

Las relaciones diplomáticas entre Argentina y Chile se profundizaron en los años siguientes. Sobre todo por la reconstrucción del Ferrocarril Trasandino, que fue nuevamente librado al servicio después de una década inactivo por el aluvión de Cacheuta. A partir de entonces se facilitaron los contactos entre presidentes, cancilleres y demás autoridades (gran impacto causaron los encuentros entre Perón e Ibáñez 
en 1953). A pesar de ello, no se alcanzaron acuerdos para reconocer las denominaciones de origen, respectivamente. En la siguiente norma legislativa Argentina insistió con su tendencia a ignorar las DO en general, y el pisco chileno en particular. En efecto, la Ley $\mathrm{N}^{\mathrm{o}} 14.878$ (1959) reiteró conceptos de la regla anterior. El artículo 17, según está consignado en la primera página del Boletín Oficial 19.095, dedicó tres incisos a delimitar los respectivos conceptos:

i) "Aguardiente de vino, pisco o brandy, el producto de la destilación especial de vino sano, cuya graduación alcohólica al salir del destilador no sea superior a setenta y cinco por ciento $(75 \%)$ en volumen de acuerdo a la reglamentación que dicte el Instituto".

En estas definiciones, la ley argentina daba un paso más en el camino de considerar como genéricos los productos con denominación de origen. Se consolidaban las DO champagne, cognac y pisco. El proceso de desconocer las DO y confundirlas con genéricos, iniciado a fines del siglo XIX, se consolidó en esta ley sancionada en 1959 y vigente hasta el momento de escribirse el presente trabajo (2013).

\section{Los pleitos con Perú}

Resulta notable el pleito sobre el pisco que todavía existe entre Chile y Perú. La industria de este país reivindica los derechos exclusivos a utilizar esta Denominación a partir de los antecedentes generados por la producción de aguardientes en la época colonial, a gran escala, para abastecer los mercados de Lima y Potosí. El uso del puerto de Pisco para el transporte de este aguardiente fue el motivo por el cual se comenzó a asociar el nombre de pisco con el aguardiente elaborado en el valle de Ica, en un proceso parecido a los vinos de Oporto, elaborados en el valle del Duero.

Junto con la fortaleza de tener el puerto de Pisco, fundado en 1640, la industria del Perú tenía sus debilidades para competir con el pisco de Chile. Sobre todo por la pérdida de continuidad que tuvo la vitivinicultura peruana. Poderosa y brillante en los siglos XVI y XVII, fue la mayor viticultura de América. Pero posteriormente la clase dirigente local perdió interés en la actividad y priorizó otras producciones como por ejemplo algodón. Además, la producción de aguardiente de caña, de menores costos, sustituyó en buena medida al aguardiente de uva. En cierta forma, la clase dirigente peruana abandonó a la industria vitivinícola y por este motivo Chile tomó la posta con relativa facilidad.

En los últimos años se ha producido un choque de intereses entre peruanos y chilenos sobre los derechos sobre el pisco. La comunidad académica peruana ha realizado importantes estudios para dar a conocer la historia de este producto (Huertas, 2004). En Chile también se han registrado trabajos de envergadura (Cortés, 2005). De todos modos, todavía hay un debate sobre el tema.

En Chile se reconoce la existencia del pisco peruano, con algunas miradas contradictorias. En el siglo XIX, Manuel Rojas en su Viticultura y Vinificación, al hablar del pisco, mencionaba que "conocido es en todo Chile el pisco de Locumba (Perú)" (Rojas, 1891: 374). Resulta notable que el citado agrónomo chileno pudiera reconocer un buen aguardiente peruano por su identificación geográfica, no así los de su propio país, al menos en ese momento. Algo parecido sucedió con el relato histórico. En Perú se suele señalar que el Pisco Sour se inventó en el Hotel Mauri, en Lima, con motivo de la visita del turista californiano Víctor Vaugn Morris a comienzos del siglo XX. Pero como ya se ha señalado, un siglo antes, ya era habitual en Chile el consumo de este producto, según el testimonio del viajero francés Lafont de Lucy (1853). Confusiones parecidas se han registrado en la literatura, como en el caso de Isabel Allende. En su novela Mi país inventado la citada escritora afirma que el pisco es el trago nacional de Chile pero a la vez "el nombre de este licor se lo usurpamos sin contemplaciones a la ciudad de Pisco en Perú” (Allende, 2003: 23).

\section{Conclusión}

El pisco representa un caso excepcional en el Cono Sur de América debido a los serios obstáculos que se levantaron para el desarrollo de las DO en la región. El principal problema fue la contaminación identitaria, introducida por los tecnócratas extranjeros especializados en el campo de la agronomía y la enología, continuada por sus pares nacionales, legalizada por el Estado y extendida por los inmigrantes. Estos grupos impulsaron el uso de las DO europeas como genéricos, para denominar productos elaborados en Chile y Argentina, a la vez que inhibieron el desarrollo de las DO locales.

Uno de los pocos casos que logró romper este círculo vicioso fue el pisco. Registrado por primera 
vez como marca en 1882, y reconocido implícitamente por el agrónomo Manuel Rojas en 1891 y René Le Feuvre en 1892. No obstante ello, el pisco siguió figurando como genérico para la ley de vinos chilena de 1916. Esta tendencia se rompió en 1931 cuando el presidente Ibáñez estableció su delimitación y protección por el DFL 181. Se produjo así el primer reconocimiento de una DO en el Cono Sur, iniciativa que se ha consolidado en la actualidad.

De todos modos, poco se avanzó en el reconocimiento regional de la DO Pisco. En Argentina, la ley de vinos de 1938 insistió con el uso de pisco como genérico, concepto reiterado en la ley de 1959 que se mantiene vigente en la actualidad. Resulta paradójico que las relaciones entre Argentina y Chile, tan activas en otros campos como el comercio, el transporte y la construcción de corredores bioceánicos, todavía no haya sido capaz de lograr un acuerdo de reconocimiento mutuo de Denominaciones de Origen.

Se nota que, a pesar del caso excepcional del pisco, todavía resulta débil el concepto de DO en Chile y en Argentina. En ambos países no se ha podido desarrollar todavía una cultura de apreciación y valoración de la relevancia que pueden tener las DO y sus efectos benéficos tanto desde el punto de vista territorial como social, pese a la importancia que su presencia reviste en el desarrollo de un conjunto de rituales claves para facilitar la integración social y fortalecer la cohesión familiar en el mundo rural, especialmente en los casos de los campesinos y arrieros, quienes, a falta de mate, consumían aguardiente con agua, limón y azúcar. En este sentido, es factible que estemos en presencia de los primeros antecedentes del Pisco Sour.

En el campo territorial, las DO contribuyen a arraigar la población al territorio rural y prevenir migración a las ciudades, acción particularmente valiosa en países con tendencia crónica a la macrocefalia (tanto Chile como Argentina concentran un tercio de la población nacional en su capital). Desde el punto de vista social, la DO apoya la pequeña propiedad y ayuda a los pequeños viticultores a lograr una rentabilidad de sus minifundios. Así se refleja en el caso del pisco, que ha permitido el florecimiento de una comunidad de 3.000 pequeños y medianos viticultores con propiedades de 3,3 hectáreas de promedio cada uno.

\section{Agradecimientos}

Artículo preparado en el marco del Proyecto FONDECYT Regular COD 1130096.

Especial agradecimiento al ingeniero agrónomo Fernando Herrera Henríquez, gerente de la Asociación de Productores de Pisco de Chile, quien ha sido un colaborador clave en la producción del presente artículo.

\section{Literatura Citada}

Allende, I.

2003. Mi país inventado. Areté. España. 221 pp.

Celestino, S.; Blánquez, J. (editores científicos).

2013. Patrimonio cultural de la vid y el vino. Universidad Autónoma. Madrid, España. 407 pp.

Cortés, $\mathrm{H}$.

2005. El origen, producción y comercio del pisco chileno, 1546-1931. Universum, 20 (2): 41-81.

Duhart, F.; Corona, S.

2010. Vinos de América y Europa. Catorce miradas desde las ciencias del hombre. Éditions Le Manuscrit. París, Francia. $352 \mathrm{pp}$.

Errázuriz Tortorelli, C.

2010. Indicaciones geográficas y denominaciones de origen. Propiedad intelectual en progreso. Revista Chilena de Derecho, 37 (2): 207-239.

Gay, C.

2009. Historia física y política de Chile. La Agricultura. Tomo I. Biblioteca Fundamentos de la Construcción de Chile. Santiago, Chile. 340 pp.
Gil, G.; Pszczólkowski, P.

2007. Viticultura. Fundamentos para optimizar la producción y calidad. Ediciones Universidad Católica. Santiago, Chile. $535 \mathrm{pp}$.

Gómez Arriola, I.

2009. El plan de manejo para el Paisaje Agavero y las Antiguas Instalaciones Industriales de Tequila. El patrimonio cultural como detonador del desarrollo regional, antecedentes, compromisos y retos. Apuntes, 22 (2): 124-141.

Hernández, J.J.

2010. El vino mescal de Tequila: entre el pule, el aguardiente de caña y el vino de uva, pp. 25-42. En: Duhart, F.; Corona, S. Vinos de América y Europa. Catorce miradas desde las ciencias del hombre. Éditions Le Manuscrit. París, Francia. 352 pp.

Huertas, L.

2004. Historia de la producción de vinos y piscos en el Perú. Universum, 19 (2): 44-61.

Huertas, L.

2012. Cronología de la producción del vino y del Pisco (Perú 1548-2010). Editorial Universitaria. Lima, Perú. 391 pp. 
Instituto Nacional de Propiedad Industrial.

2010. Historia gráfica de la propiedad industrial en Chile.

Pie de Texto Consultores y Editores. Santiago, Chile. 200 pp.

Lacoste, P.; Muñoz, J.G. y Castro, A.

2010. Variedades de uva en Chile y Argentina (1550-1850).

Genealogía del Torrontés. Mundo Agrario, 10 (20): 1-36.

Lacoste, P.; Yuri, J.A.; Aranda, M.; Castro, A.; Quinteros, K.;

Solar, M.; Soto, N.; Gaete, J. y Rivas, J.

2014. Aguardiente y viñas en Chile: Quillota, Colchagua y Cauquenes en el padrón de 1777. Inédito. Santiago, Chile. $200 \mathrm{pp}$.

Lafond de Lucy, G.

1911. Viaje a Chile. Traducido de la edición francesa de 1853 por Federico Gana. Imprenta Universitaria. Santiago, Chile. $217 \mathrm{pp}$.

Le Feuvre, R.F.

1892 (5 y 20 de julio). Informe relativo a los datos estadísticos recibidos y al estudio practicado, en vista de la publicación de una obra especial sobre la industria vitícola de Chile en las provincias de Tarapacá, Antofagasta, Atacama y Coquimbo, que forman la región norte de la República, pp. 335-354. En: Sociedad Nacional de Agricultura. Boletín de la Sociedad Nacional de Agricultura (BSNA).
Vol. XXIII. Sociedad Nacional de Agricultura. Santiago, Chile. 748 pp.

Le Feuvre, R. F.

1892 (5 y 20 de octubre). La industria de las pasas en Chile, pp. 597-607. En: Sociedad Nacional de Agricultura, Boletín de la Sociedad Nacional de Agricultura. Vol. XXIII. Sociedad Nacional de Agricultura. Santiago, Chile. 748 pp.

Poças Pintão, M.J.

2012. Fraudes, imitações, apropiações da imagen do Vinho do Porto, pp. 441-446. En: Barros Cardoso, Antonio, Trilho, Silvia (coordenaçaõ). I Congresso Internacional Vinhas e Vinhos. Actas. Câmara Municipal de Viana do Castelo. Porto, Portugal. 632 pp.

Pszczólkowski, P.; Ceppi de Lecco, C.

2011. Manual de Microvinificación. Guía práctica para la elaboración de vinos. Ediciones Universidad Católica. Santiago, Chile. 122 pp.

Ribeiro de Almeida, Alberto.

2010. A Autonomia Jurídica da Denominação de Origem. Uma perspectiva transnacional. Uma garantía de qualidade. Coimbra Editora. Coimbra, Portugal. 1475 pp.

Rojas, M.

1891/1950. Tratado de viticultura y vinificación. $1^{\text {a }}$ edición. Imprenta de la Libertad. Talca, Chile. 1891, 667 pp.; $5^{\text {a }}$ edición. Nascimento. Santiago, Chile. 829 pp. 